\title{
Germanica
}

GERMANICA $48 \mid 2011$

Max Frisch philosophe?

\section{Existence, altérité et transcendance : Max Frisch philosophe}

Existenz, Alterität und Transzendenz: Max Frisch als Philosoph.

Existence, otherness and transcendence : Max Frisch as a philosopher.

\section{Régine Battiston}

\section{(2) OpenEdition}

Journals

Édition électronique

URL : http://journals.openedition.org/germanica/1179

DOI : 10.4000/germanica. 1179

ISSN : 2107-0784

Éditeur

Université de Lille

Édition imprimée

Date de publication : 1 juillet 2011

Pagination : 33-54

ISBN : 9782913857278

ISSN : 0984-2632

\section{Référence électronique}

Régine Battiston, «Existence, altérité et transcendance : Max Frisch philosophe », Germanica [En ligne], 48 | 2011, document 3, mis en ligne le 01 juin 2013, consulté le 06 octobre 2020. URL : http:// journals.openedition.org/germanica/1179; DOI : https://doi.org/10.4000/germanica.1179

Ce document a été généré automatiquement le 6 octobre 2020.

(c) Tous droits réservés 


\title{
Existence, altérité et transcendance : Max Frisch philosophe
}

\author{
Existenz, Alterität und Transzendenz: Max Frisch als Philosoph. \\ Existence, otherness and transcendence: Max Frisch as a philosopher.
}

Régine Battiston

1 L'œuvre de Max Frisch se développe sur le terreau de l'existentialisme de Kierkegaard avec lequel elle prend ses distances pour s'épanouir dans l'identité (narrative) d'un existentialisme heideggérien finissant. À la recherche de leur Moi, les personnages du monde littéraire de Frisch se découvrent une identité plurielle d'être en devenir. Le fait d'être pour l'Autre, de le chercher, de le rencontrer, d'échouer aussi dans sa relation à l'Autre féminin, montre un sujet en quête de lui-même et de sa propre identité, dans la seule voie de vie qu'est le chemin difficile à deux et en pointillés aussi. À ce sillon de la relation à l'Autre s'ajoute dans l'œuvre de maturité une conscience de citoyen engagé dans la vie et la société de son temps. L'œuvre tardive, dans une interrogation récurrente sur le vieillissement et la mort et de fugaces images de transcendance dans un après-vie en dehors du religieux, s'éloigne du sentier du concept de l'identité, pour envisager l'altérité autrement que dans un face à face difficile avec l'autrui féminin.

2 L'œuvre de Frisch s'inscrit globalement dans trois grandes phases, qui vont des relations amoureuses et éphémères, à l'engagement citoyen et enfin au pessimisme et à la désillusion, qui est présentée à travers les méditations de la fin de l'œuvre. Toute la réflexion de Frisch est basée dans la première moitié de son œuvre sur l'exemplarité dans un monde romanesque ou théâtral. La fiction lui permet de constater les travers des relations interpersonnelles et de faire en sorte que le lecteur soit en mesure d'en tirer des leçons, sans que celles-ci ne soient édictées. Dans la première partie de l'œuvre, les thèmes que sont la recherche de son identité, l'amour des femmes et les difficultés de cette relation de couple, sont déclinés surtout dans les romans. Dans la deuxième partie de son œuvre, dans une ouverture au monde et à la société qui l'entoure, Frisch s'engage de manière citoyenne dans des voyages, des discours, des 
tribunes et prises de décisions publiques, que son statut d'écrivain reconnu lui permet ; il intègre alors dans son œuvre les constats qu'il fait des problèmes dans le monde. La troisième partie de sa vie littéraire est marquée par la désillusion et le pessimisme, qui orientent son œuvre vers les thèmes de la vieillesse et de la mort et elle culmine dans le discours des Journées Littéraires de Soleure de mai 1986. Si on attend plus d'apaisement de la part d'une œuvre de la vieillesse, celle de Frisch ne passe pas de l'inquiétude à la quiétude, les traces de métaphysique et de transcendance restent ténues, Frisch reste ancré dans le présent de son temps.

3 Les thématiques de l'identité et de l'altérité placent son œuvre aux confins de la réflexion philosophique. Si Frisch refuse de s'appliquer le terme de "philosophe $»^{1}$, il pense à un philosophe qui développe des théories dans un domaine abstrait et/ou qui donne des leçons à ses lecteurs. Dans ce sens là, il a raison de dire qu'il ne s'est pas comporté comme un philosophe, ou comme un donneur de leçons moralisant. Mais il n'en est pas moins un homme d'éthique et un penseur soucieux d'autrui, de son devenir, de celui de la société et du monde dans lequel il vit; et de ce point de vue éthique, il émet des pensées philosophiques, contenues dans ses œuvres romanesques et théâtrales. S'il refuse de passer pour un donneur de leçons et d'être taxé de philosophe, on peut lui rétorquer qu'il ne faut pas nécessairement se dire ou s'ériger en philosophe pour faire de la philosophie. L'écrivain Frisch refuse toutes les étiquettes, tout ce qui contraint et qui fige l'être dans un moule ou un concept. Il a appliqué ceci à toute son œuvre et la conséquence est la première vertu du philosophe d'après Kant...

\section{Existence et identité}

4 À travers toute l'œuvre de Frisch est filée la relation à l'Autre Féminin. Elle constitue la relation de base de tous ses personnages depuis le début de l'œuvre (Jürg Reinhardt, 1934) jusque dans les années 1960, où elle atteint son point culminant, pour rester présente, mais de manière moins soutenue dans l'œuvre tardive. Dans Homo faber (1957), à travers le personnage de Walter Faber, c'est un monde masculin, orienté par des tendances de chauvinisme mâle (le terme est de Frisch dans Montauk), de machisme qui se déploie ; ce monde basé sur le non-dit et l'exposition au regard de l'Autre (versus regarder l'Autre, cf. Sartre) constitue le terreau des relations homme-femme. Cette relation homme-femme n'est pensée que dans un jeu de séduction et de conquête, qui aboutit à une vie quotidienne domestique, dont l'échec est inéluctable. Cette relation à l'Autre féminin est une mise en scène qui sert au questionnement de l'être sur sa finalité ici bas, son altérité, sa capacité (ou son incapacité) à vivre en harmonie avec autrui, à se développer avec et aux côtés d'autrui, en assumant ses angoisses existentielles, ses faiblesses, souvent découvertes à travers ou par le biais de l'échec de la relation du couple.

Dans le monde des relations homme-femme chez Frisch, la jalousie tient un grand rôle, dans un univers où le face à face et le dialogue occupent très peu de place et où la fantasmagorie masculine édictée par un narrateur du même sexe voit la femme comme une partenaire de vie, sans chercher à la comprendre, sans chercher à entrer dans son monde féminin, aux codes stéréotypés. C'est le regard seul qui pousse le partenaire à douter de sa compagne, dans un monologue obsessif avec lui-même, dans un doute sur ses propres capacités de séducteur et de compagnon de vie agréable ; jusqu'à ce que le pas franchi, du doute vers la vérification des angoisses qui le tenaillent, dévoile une 
névrose obsessionnelle provoquant une crise conjugale et l'échec d'un couple (cf. première scène de Mein Name sei Gantenbein dans l'appartement vide).

6 L'aveuglement envers les autres et surtout envers les femmes de sa vie souligne une position de suffisance qui culmine dans le péché d'orgueil : il s'agit d'un aveuglement face aux évidences, à la profondeur de la vie, au hasard et à la coïncidence, aux aléas de la vie. Les personnages masculins sont sans respect et affection sincèreenvers l'autre féminin (surtout Faber). Le regard, sa problématique (cf. Sartre) et ce qu'ils impliquent sont théorisés dans le Journal 1946-1949 sous forme de variation du troisième commandement du décalogue que Frisch transforme en: «Tu ne te feras pas d'idole, est-il dit de Dieu. Cela ne paraît-il pas aussi valable en ce sens : Dieu, c'est-à-dire ce qui est vivant en chaque homme, ce qui est insaisissable? $»^{2}$. Ce commandement si important dans l'œuvre de Frisch (pour Ludwig Anatol Stiller ou aussi pour Andri dans Andorra) est remis en question par le comportement des héros masculins envers leurs compagnes et c'est Stiller qui exprime le mieux la difficulté de voir l'autre tel qu'il est ou voudrait se montrer, sans le faire entrer dans un cliché ; renouveler son regard sur autrui, accorder à l'autre son autonomie de sujet agissant et pensant (cf. Ronald Laing, " interpersonal perception $»^{3}$ ), telle est la gageure impossible. Stiller a beau stipuler que "Toute image est un péché. C'est exactement le contraire de l'amour, vois-tu, ce que tu fais là avec de tels discours $»^{4}$, il commet ce péché d'orgueil et de perception d'autrui à travers des pré-requis, issus de la vie quotidienne : chacun n'étant en fin de compte capable de percevoir l'autre qu'au travers de son propre Moi et les critères du regard personnel ne sont pas renouvelables à l'infini et au gré des envies. Chaque conception du monde, même si elle évolue, reste rivée à sa propre conscience des autres et des choses. Frisch montre à travers les destins de ses personnages masculins que la maxime de Kierkegaard : «Quand j'ai compris un autre sujet, sa réalité est pour moi une possibilité $»^{5}$, est difficile à prendre en compte et à appliquer, car ladite possibilité évolue dans le temps, l'autre sujet et le propre Moi aussi (cf. l'individu dans le fleuve d'Héraclite).

$7 \quad$ Frisch joue aves les trois stades de Kierkegaard, en tenant compte de la Geworfenheit de Heidegger (être jeté là dans le monde, qui débouche sur l'angoisse existentielle) et avec la notion de choix de vie de Sartre. Il démontre que les théories philosophiques et le niveau psycho-sociologique de la vie ne coïncident pas $^{6}$ - et que le plus important est la vie elle-même (voir la fin de ses romans). Ses personnages masculins sont surtout des observateurs de la vie qui ne veulent pas prendre position pour pouvoir conserver la possibilité de changer de cap, de vivre de nouvelles expériences, qui par définition laissent toute possibilité de choix ouverte. La philosophie n'est donc pas la vraie vie (la littérature non plus, finalement). Il affirme, dans un entretien avec Heinz Ludwig Arnold, qu'il tire ses exemples de la vie et ne cherche pas à la théoriser : «[...] En fait je ne viens pas de la littérature, mais de l'expérience personnelle et je me placerais, si le terme n'est pas usurpé, dans la catégorie des écrivains qui écrivent par légitime défense $»^{7}$. Si bien que son œuvre se veut être uniquement l'illustration et/ou le refus de théories philosophiques, qui peuvent lui servir d'arrière plan de référence.

8 Si le regard de l'autre fige l'être dans sa position de compagnon de vie, Frisch souligne que le fait de se supporter et de supporter les autres échappe à l'existentialisme kierkegaardien, qui prône que la répétition des actes et des événements de la vie est inévitable dans un quotidien de vie (même dans une vie de solitaire) et nécessaire pour l'amélioration de soi. Si Stiller veut échapper à la nécessité de la répétition, alors que 
dans la société il est confronté en permanence à cette répétition, il combat une évidence. Et il échoue devant la force de cette évidence. La relation à autrui débouche sur le triomphe de l'enfer de la vie commune, impossible lieu d'épanouissement pour le personnage masculin. L'enfer du face à face et du quotidien est motivé par le déni des responsabilités, les personnages masculins de Frisch ne veulent pas être père, ils ne veulent pas de continuité, de succession, ils veulent le moment unique, vivre l'instant et se cherchent à travers l'autre et l'absence de traces laissées derrière soi. Mais la recherche d'un idéal et les tentatives d'évitement se heurtent au quotidien, aux limites imposées par la vie, le milieu social, les capacités de son propre Moi. Les personnages de Frisch se cognent sans cesse à ces limites, en voulant les outrepasser, les dépasser ou les ignorer (cf. Stiller). La vie leur apparait de ce fait comme une prison, leur questionnement sur la vraie vie, son sens, la vie dans l'instant et la non-répétition de situations vécues les ancre dans l'existentialisme, dont ils interrogent les multiples facettes. La conséquence de cette interrogation est la mélancolie que frôlent certains (Faber, Stiller, entre autres). Ils constatent l'incapacité des valeurs bourgeoises à prendre en compte les attentes du Moi, l'individualité de chacun, ses aspirations et ses contrariétés. Frisch fait une lecture difficile du marxisme, qui demande le développement de chacun en liberté dans la société, et de l'existentialisme sartrien, qui affirme que chacun est responsable de son développement et qu'il devient ce qu'il veut. Son expérience de la vie lui montre cependant que la conviction sartrienne du " choisis-toi toi-même » et "deviens ce que tu dois ", ne fonctionne pas, car l'être est soumis avant tout au milieu dans lequel il naît et grandit, qui conditionne lourdement son avenir et ses choix de vie.

Refusant de donner des leçons, Frisch base son œuvre romanesque sur la multiplication de scénarios de vie (essais, variations sur ces essais, représentations et échecs aussi). Il ne commente et/ou ne conclut jamais (aucun de ses personnages non plus) en tirant une leçon de ces variations de scénarios de représentations de la vie, de tranches de vie. Il livre au lecteur des scénarios qui semblent au premier abord disposés au hasard, de manière anarchique et sans lien entre eux (là où tout est calculé par cet architecte du texte) $)^{8}$. Il n'esquisse aucun commentaire, se contente de juxtaposer des scénarios, parfois avec un changement de lieu, de personnage, bref sans lien avec ce qui précède (et qui suivra). Pourtant s'il n'édicte jamais de sentence, son agencement (peu anarchique) de scénarios de vie est renforcé par l'insertion de fables dans le récit (cf. Stiller, Mein Name sei Gantenbein), qui font office de conclusion aux démonstrations que la narration, à travers l'agencement des scénarios de vie, suggère. Ces fables viennent souligner ou renforcer le caractère de leçon qui se dégage à la lecture des scénarios épars. Ainsi Frisch, se défendant de vouloir donner des leçons, contourne-il par le biais de la fable, ce dont il se défend. La fable fait office de dernier écho, suite au déferlement de scénarios de vie qui mènent tous à l'échec du couple, elle constitue l'illustration ultime de ces échecs, tel un point sur le « $\mathrm{i} »$. Sur un ton ironique parfois, anodin ou amusé, ces fables donnent à réfléchir au lecteur, en formalisant de manière courte et condensée, sur le problème soulevé et récurrent : un couple est voué à l'échec, même dans la littérature (un homme aime une femme qui aime un autre homme, cf. Mein Name sei Gantenbein). La solution du romancier qu'il est, consiste à mettre l'amour en histoires et dans le roman Mein Name sei Gantenbein, il fait appel à des variations diverses pour montrer les écueils qui guettent l'amour, dont la jalousie (fable du boulanger, d'Ali et Alil, de Philémon et Baucis). Si Frisch montre par des illustrations et suggère en montrant des scénarios qui s'enchaînent et qui débouchent toujours sur le 
même constat d'échec (y compris dans la fable), son procédé d'inclure des fables en guise de sentence fait de sa méthode, de facto, un développement moral à portée philosophique, une approche philosophique, en somme, même s'il se défend de ne pas y toucher (avec le leitmotiv qui lui tient à cœur, de ne pas vouloir figer les gens dans un moule d'où ils ne sortiront plus). Les fables font office de paraboles, Frisch les appelle lui-même ainsi ${ }^{9}$. Dans un sens biblique et moral, ces paraboles sont doublées de critique sociale (Stiller, par exemple), quant elles ne sont pas mises en écho, par une sorte de double couche, par le mythe (Don Juan, Hermès, entre autres).

Dans le cheminement de son œuvre se dégage rapidement et de manière définitive le pessimisme de Frisch à propos de la capacité d'amendement de l'être humain, sa capacité de se remettre en question et de se transformer. À travers des personnages désillusionnés et parfois immoraux (exemple Schaad dans Blaubart), il creuse le sillon de la notion d'échec du couple, de la relation avec l'autre féminin, également à travers les tentatives de suicide de plusieurs de ses héros. Ils sont figés dans l'incapacité chronique d'une vie commune, dans le fait de ne pas parvenir à aller au bout de leurs intentions (par exemple, Stiller ne parvient pas à tuer quelqu'un pendant la guerre d'Espagne). Frisch met admirablement à nu à travers toutes ces variations de scénarios de vie, les mécanismes qui mènent à l'échec du couple et de la vie commune. L'échec relationnel avec l'autre féminin transparaît paradoxalement dans les vertus des dialogues, où le non dit permet aux personnages masculins de ne pas affronter la réalité d'une relation déliquescente avec leur partenaire féminin (cf. Homo faber ${ }^{10}$ ). Une autre forme de la relation (devenue) bancale est la cécité ou le refus de voir les faits, de les reconnaître (encore Homo faber ${ }^{11}$ ).

11 Chez Frisch, l'amour va aussi de pair avec l'estime de soi et la recherche de soi à travers le regard du partenaire féminin. Son œuvre dénote une recherche sous forme de compulsion de répétition de la femme aimée, des femmes séduites et conquises et ensuite quittées. Cette recherche est couronnée par un constat sur sa vie amoureuse (pour le moins littéraire) par le reproche que lui fait sa mère, qu'il livre dans Montauk: "Tu ne devrais pas toujours écrire sur les femmes, car tu ne les comprends pas $»^{12}$. À cette constatation restituée par un fils lucide font écho les regrets dudit fils à propos de ses femmes (Ingeborg Bachmann, Marianne sa presque ex-épouse au moment de la rédaction du livre) sur ses histoires d'amour échouées. Le temps fait son œuvre de réflexion et de sape, la relation féminine et la figure de la femme se font plus ténues ou deviennent amères dans l'œuvre tardive (Triptychon, 1978, Blaubart, 1982, il est à noter la quasi absence de personnage féminin dans Der Mensch erscheint im Holozän, 1979). Le renoncement à la passion amoureuse se fait au détriment de la présence de la femme ou d'un autre partenaire (l'amitié apparait peu dans l'œuvre de Frisch, il la revendique seulement pour sa propre vie); le renoncement au face à face avec l'alter ego féminin débouche dans la solitude, dans un face à face avec soi-même.

12 La recherche permanente de Frisch à travers ces figures de femmes et d'une relation à l'autrui féminin se fonde et est motivée par une angoisse existentielle de l'être dans le monde. Frisch prend ce concept au pied de la lettre et l'exploite sous différents aspects, comme le fait de s'inscrire dans une réalité et un territoire, une histoire, une société, en face à face avec l'autrui féminin. Son œuvre met l'accent sur l'être et ses possibilités, les explorations de ses possibilités. L'existence ne relève pas seulement de ce que fait l'être ou de ce qui lui est donné, mais de toutes les possibilités non exploitées, les manques, les échecs. Comme le souligne Fabien Chareix, «l'existentialisme, qui clôt une période 
de l'histoire de la philosophie ouverte sur la problématique de la valeur de l'existence, promeut la catégorie de l'existentiel comme détermination des conduites proprement humaines $\aleph^{13}$. Frisch illustre dans son œuvre la difficulté de l'être à comprendre le monde et l'existence et de vivre cette existence dans la détermination de l'existence humaine par elle-même. Il démontre aussi comment l'homme cherche à se situer dans le monde pour penser. Il souligne par le destin fractionné de tous ses personnages, la difficulté de l'homme de comprendre et de concevoir sa façon d'être au monde. Car selon Sartre, l'homme est libre de décider de son destin, libre de ses actes et de ses choix. Or les personnages de Frisch se heurtent à des limites telles les rencontres, le milieu social qui induit le destin de chacun, le caractère de leur personne aussi - Frisch démonte implicitement la théorie de Sartre; même dans le domaine du mythe (cf. Don Juan), il bat les certitudes en brèche et met les théories philosophiques en difficulté. Il montre à travers ses personnages les possibilités offertes, mais aussi les limites de chaque être, à commencer par celles dans sa personne, son milieu, le déterminisme de celui-ci, de l'époque et de la société dans laquelle l'individu est jeté (on retrouve ici des aspects de la " Geworfenheit » de Heidegger).

L'œuvre de Frisch peut certes se lire à plusieurs niveaux et de nombreux critiques (et non des moindres ${ }^{14}$ ) l'ont largement prouvé. Entre le niveau premier de la fable et celui de l'intertextualité philosophique, l'éthique de Frisch transparaît sans cesse, une éthique soucieuse de l'homme dans sa chair, sa vie privée, ses relations à autrui et au monde. L'homme reste au centre de ses préoccupations. Les vertus ou les manques de ses héros soulignent cette quête dans un monde frischien sans polarisation, où les limites entre le bien et le mal, la vérité et le mensonge, la responsabilité, le hasard, la coïncidence, la fatalité sont floues. Comme chez Saint Augustin l'espace terrestre est le lieu du quotidien, de ses passions (et désillusions) et du souci de soi. Le premier niveau de lecture au niveau de perception de la réalité du monde par ses personnages, laisse à croire à la possibilité d'une liberté de choix que les échecs permanents de tous ses personnages battent en brèche. Au niveau de l'action il y a aussi deux temps, celui de la révolte des héros contre la société et ses lois trop rigides (Don Juan, Kürman, Stiller, Walter Faber) et l'étape de la résignation, à travers la solitude ou la maladie et la mort, l'acceptation et la soumission, en guise de fatalisme.

La problématique de l'altérité a constitué le point central de l'œuvre de Frisch jusque dans les années 1970, ceci se vérifie tant dans les romans que dans les œuvres théâtrales. Longtemps cette altérité, uniquement centrée sur celle féminine et le face à face avec autrui, s'inscrivait dans une relation de couple (éphémère, certes) sans cesse renouvelée et reconsidérée. Nous soulignons que :
l'univers littéraire de Frisch montre un monde complexe lié à des conflits, des désillusions, des déceptions, des personnages en devenir qui se cherchent et dont les interrogations sur le sens et la trajectoire de leur vie, sont une preuve de leur fragilité, mais aussi de leur possibilité à se transformer. Toute l'œuvre de cet écrivain montre finement la tension entre réalité et possibilité, le problème de l'identité y étant central ${ }^{15}$.

Telle une compulsion de répétition dans l'échec d'une impossible vie commune, la relation à l'autre féminin atteint un sommet dans les œuvres de Don Juan et surtout Blaubart. La fin de ce récit résume d'ailleurs la conviction de Frisch, déclinée à travers toutes ses œuvres et culminant dans le personnage de Felix Schaad (le prénom sonne 
comme un bonheur impossible) : l'homme se cherche dans sa compagne à travers une relation qui n'est pas destinée à durer. Jamais il ne s'inscrit dans une ouverture vers l'autre, il craint le regard de l'autre et ne parvient pas à comprendre ses compagnes, il n'atteint pas l'empathie nécessaire et salutaire dans un couple - c'est d'ailleurs ce que constate amèrement le juge pendant le procès, en examinant sa prétendue culpabilisé dans le meurtre d'une de ses ex-épouses Rosalinde: Schaad ne comprend pas ses épouses ${ }^{16}$. De ce fait toutes les œuvres débouchent sur un scepticisme quant à la relation avec autrui et sur une résignation à vivre, à profiter de l'instant (cf. la fin de Mein Name sei Gantenbein). Le doute et la déception qui transparaissent dans la fin du roman Stiller ("Stiller resta à Glion et vécut seul»), montrent le concept de l'altérité comme une recherche permanente de soi dans l'autre, sans parvenir à accepter l'autre dans sa différence. Est toujours mis en scène un homme en devenir certes, mais un homme sans avenir et sans illusions, qui reste cloîtré dans sa solitude, car comme le constate Schaad: il n'y a pas de conscience commune ("Es gibt kein gemeinsames Gedächtnis“" $\left.{ }^{\text {“17}}\right)$.

\section{Altérité et engagement}

16 Frisch vit dans l'époque des années 1950-60 où de nombreux intellectuels se sont demandés comment écrire et/ou philosopher après Auschwitz ? C'est aussi la réflexion d'Hanna Arendt sur la mission de la philosophie, comment la réformer pour qu'elle puisse réintégrer sa mission éthique. C'est sur ce plan que l'œuvre de Frisch rejoint, à partir des années 1970 tout au moins, les préoccupations de certains philosophes de son temps, lorsque la veine existentialiste s'estompe au profit de l'engagement politique qui débouche dans le structuralisme et préfigure le postmodernisme. Frisch est d'accord avec Arendt, pour dire que c'est dans l'engagement et le soutien à l'action politique, que réside une partie de la mission d'un intellectuel (cf. Les Origines $d u$ totalitarisme, 1951); il est sur ce plan sur la même ligne que Sartre. Ainsi passe-t-il à partir des années 1960 à des activités ou des écrits politiques et à des actions de terrain jusque dans les années 1980 ; son engagement trouve son summum dans les années 1970 (à partir de la Guerre Froide et de celle du Vietnam), de mai 68 et dans les années de terrorisme. Sa critique de la société de consommation et des médias (déjà fustigés par Adorno), dans les textes en prose, est l'amorce d'un virage vers plus d'éthique politique. Cette attitude devient nette dans les textes publics, les discours depuis le début des années 1960, particulièrement ceux des prix littéraires.

Apporter son soutien aux opprimés (aux Chiliens, par exemple), se positionner contre la Guerre au Vietnam, s'en prendre à la défection et à la passivité de son propre pays, voire à son laxisme ou à sa complaisance, sa mission s'amplifie avec le temps (cf. le discours Schweiz als Heimat?, "Großer Schillerpreis der Schweizerischen Schillerstiftung“, 1974). Il fait des discours de vérité, étayés par un entendement éclairé, à des fins d'éclaircissement; sa dénonciation du mensonge politique, du nondit, de la Raison d'état, de l'amnésie pour le passé de collaboration nazie est unilatérale, elle va engendrer sa mise sur écoute par les renseignements généraux suisses, ce qu'il ne découvrira que dans les années 1980. La tension permanente entre lui et son pays, la Suisse, est devenue un état de fait. Son exigence morale d'équité lui fait constamment tenter de secouer la conscience de ses concitoyens, comme le fit aussi son collègue Durrenmatt, dans un autre style satirique. 
18 À partir des années 1950, on sent dans son œuvre un penchant de plus en plus marqué pour l'avenir de l'humanité, qui s'accompagne d'un élargissement de sa pensée, de la sphère des relations homme-femme à celle de la société qui l'entoure. Ses discours à partir de cette période sont marqués par l'idée dialectique de guerre et de paix, ils dénotent une dénonciation de la guerre, mais contiennent peu de signes d'espoir de paix (messages sans illusion), mais plutôt des propositions qui vont vers la paix. Sur le ton de la rébellion et de l'indignation, il attaque souvent (et l'avenir lui donnera raison) le système capitaliste dans son ensemble et le milieu des banques et des affaires (dont ceux de la Suisse). Ses prises de position pourraient être le motif de développer des théories politiques (de type marxiste, par exemple), ce à quoi il ne se résout jamais. Il souligne et dénonce des cas pratiques, en observateur impitoyable de son temps. Ces chroniques politiques d'un temps passé pas si lointain, ressemblent aujourd'hui, pour une jeunesse en quête de vitesse et de télétransmission, à un message philosophique suranné. Il n'empêche qu'à l'heure de la mondialisation les analyses fines et incisives du maître sonnent encore juste, pour ceux qui ne pratiquent pas l'amnésie envers le passé.

Une des questions centrales inhérente à tous ces écrits est celle de l'efficacité de l'écriture comme arme morale. Son questionnement contre la guerre pour plus d'humanité partout sur la planète culmine dans son engagement pour une Suisse sans armée (Volksabstimmung: "Volksinitiative für eine Schweiz ohne Armee und für eine umfassende Friedenspolitik“, 1989). Son programme est fondé sur le concept de «responsabilité » d'autrui, il dénonce ainsi l'injustice, le chômage, le racisme et la bêtise (qu'il appelle l'ignorance), dans le contexte socio-politique d'après-guerre et de Guerre Froide; tout ceci avec la méthode d'un homme de plume, qui manie le verbe comme un des grands maîtres du $\mathrm{xx}^{\mathrm{e}}$ siècle. Son ami Gottfried Honegger dans son livre Widerstand aus Verantwortung (1991) lui rend un très bel et très juste hommage dans le chapitre qui lui est consacré "Wortwahrheit - Nachruf auf Max Frisch":

Des mots. Tout ce qu'il voyait se transformait chez lui en mots. Tout ce qu'il entendait devenait des mots. Ses mots ont des odeurs. Ils sont tangibles - palpables. Tous ses sens étaient orientés vers les mots. Il pensait en mots. Il respectait les mots. Il vérifait la particularité et le sens de chaque mot. Il connaissait ses mots. Il avait vécu avec et à travers eux ${ }^{18}$.

Son discours de 1958 à l'occasion du Prix Büchner (voir "Emigranten“, Rede zur Verleihung des Georg-Büchner-Preises - Gesammelte Werke, IV, 229-243) est le début dithyrambique d'une série de discours montrant son indignation et sa révolte contre l'ordre politique sclérosé et le capitalisme de rentiers. Le texte sur les émigrants italiens qui viennent travailler en Suisse, montre un pays où ils vivent au ban de la société et y sont juste tolérés ("Wir haben Arbeitskräfte gerufen und es kommen Menschen"). Son credo pour une Suisse plus humaine et moins raide est décliné sur différents modes; il attaque déjà la Suisse d'après-guerre comme étant un pays de cocagne plein de vices (1946: "Das Schlaraffenland, die Schweiz" (II, 312-318 München, Ostern 1946). Il s'en prend aussi au passé suisse, à son attitude pendant la Deuxième Guerre mondiale $(\mathrm{V}, 370-373$ - 1965) et la dénonce comme un pays sans imagination (notamment sur le plan architectural) et sans utopie (IV, 258-259), donc sans vision d'avenir. Son engagement ne se cantonne pas seulement à l'écriture, il prend position pour les Chiliens en écrivant une lettre au président du Conseil Fédéral à Berne : "Offenen Brief an den Schweizerischen Bundesrat" (Berlin, 2. 3. 1974, VI, 519-521). Sur un ton incisif et ironique, il critique la position timorée et passéiste de la diplomatie suisse et remet la compétence et la réputation de l'ambassadeur en 
question. Son combat pour une Suisse plus éthique culmine dans le discours "Die Schweiz als Heimat? Rede zur Verleihung des Großen Schillerpreises"(1974, VI, 509-518). Il passe en revue les différentes définitions du mot "Heimat", en ne reconnaissant pas la Suisse du mensonge comme étant sa patrie. On retrouve son engagement pour la liberté de pensée et de parole dans son soutien aux étudiants de l'université de Zurich en avrilmai 1968, au moment d'une réforme très contestée de l'université en Suisse (cf. "Jemand hat sich geirrt", VI, 473-475, 1968).

21 Malgré son engagement et les voyages avec Helmut Schmidt qui vont lui permettre de se profiler encore davantage comme un écrivain engagé à gauche, il va perdre ses illusions sur la vie et la permissivité en URSS (dans ce contexte il accorde son soutien à Andrej Sacharow). Ses écrits très marqués par le mot "démocratie " aux accents parfois marxisants, dénoncent aussi le commerce mondial (et suisse) des armes, la censure de la presse et notamment celle de la télévision. Les maîtres mots des années 1970-1980 sont « indépendance, liberté de conscience et d'expression, égalité devant la loi et tolérance $»^{19}$ (voir entre autres ses réflexions sur le socialisme : VI, 503, 1971).

Cette deuxième étape de son œuvre est faite d'un engagement citoyen dans le cadre d'une pensée altruiste de type socialiste. Cet engagement citoyen et politique se fait jour à partir du milieu des années 1960 ; c'est une autre forme d'altérité qui dévoile un écrivain qui s'ouvre au monde, en Suisse, à l'étranger et sur d'autres continents. C'est la période de la vie de Frisch la plus engagée dans les luttes de son temps, mai 1968, la Guerre au Vietnam, les graves exactions en Amérique du Sud, en Grèce, etc. Les écrits se transforment, de la fiction on passe à des discours, des pamphlets, le Deuxième Journal est la preuve de son questionnement permanent, de son engagement aussi, même sur le plan politique (voyage en Chine avec le Chancelier Helmut Schmidt, rencontre à la Maison Blanche avec Henry Kissinger, entre autres). Ses discours et ses petites proses reproduisent le cheminement de sa pensée à travers un engagement dans des tribunes et des voyages privés ou politiques, jusqu'à la désillusion des années 1980-1985. Max Frisch a utilisé de manière très adroite les tribunes des nombreux prix littéraires qui lui ont été décernés pour faire passer ses messages de type politiques, dont la plupart ont une forte connotation morale, sans que l'écrivain ne se réclame jamais de cette veine de pensée.

L'œuvre de Frisch se situe donc entre l'engagement littéraire et politique dans laquelle les petites proses fonctionnent comme un journal d'atelier (cf. Kleine Prosaschriften), un carnet de bord du quotidien de la réflexion d'un homme dont l'activité principale était d'écrire (il lui consacrait plusieurs heures chaque jour). Ces fameux écrits, tout comme ses nombreux discours dénotent un souci d'éthique citoyenne et d'altruisme très marqué. Il ne se base pas sur des vertus sociales ou individuelles, mais utilise sa technique classique qui est de donner des exemples précis sortis de son vécu personnel pour démontrer et convaincre; ainsi il renonce aux théories de tous bords (qui ne l'intéressent pas) et souligne toujours le manque de vertu de ses héros de fiction et aussi l'impossibilité d'atteindre quelque vertu que ce soit dans la société de son temps.

\section{Désillusion et transcendance}

Malgré son engagement politique, Max Frisch garde une conscience aiguë de la finalité de son être, de la fugacité de la vie, du temps qu'elle dure. Très tôt les thèmes de la mort et de la vieillesse marquent son œuvre et deviennent plus présents dans l'œuvre 
de la maturité et surtout celle tardive. C'est la conscience immédiate de soi, du vécu intentionnel, qui est à la base de l'être dans l'œuvre de Frisch, cette conscience lui permet aussi d'appréhender la temporalité du temps qui échappe à tout acte de représentation, notamment dans Der Mensch erscheint im Holozän. La thématique de la vieillesse y est prédominante, tant par les préoccupations que par les activités du personnage et des signes certains de sénilité se font jour. Derrière le problème de la sénilité réside celui de la fin de vie, un thème cher à l'écrivain depuis une dizaine d'années. Il met l'homme démuni en position de solitude extrême et d'impuissance, face à son destin et à la toute-puissance de la nature. La diminution de ses facultés mentales pose la question du devenir d'une humanité dont le savoir médical est de plus en plus grand, mais dont l'efficacité des progrès est parfois discutable. Si le thème de la mort est antérieur à celui de la vieillesse dans son œuvre, il gagne en intensité à partir des années 1970, où la hantise de la décrépitude, de la maladie incurable et de la mort poussent Frisch à un militantisme dans une association pour le droit au suicide qu'il évoque dans son Deuxième Journal, Tagebuch 1966-71 (1972).

La préoccupation de la transcendance est arrivée tard dans l'œuvre de Frisch et l'évocation de ce sujet ou ses allusions à ce thème sont faibles, en fonction de l'œuvre dans laquelle ils apparaissent. Dans la pièce Triptychon le dialogue entre les morts n'offre pas de signe de transcendance outre le fait que la morale issue de ces dialogues est terrestre, les hommes n'apprennent rien de ou par la vie. Le clivage homme-femme est toujours présent avec son lot d'incompréhension mutuelle. La pièce accorde une grande place au face à face impossible et insupportable homme-femme dans la vie terrestre et à ses échecs répétés. La maladie est évoquée sous plusieurs formes et reste un leitmotiv important. Cette pièce aux accents très pessimistes montre l'homme prisonnier de son destin au-delà de sa mort, dans une compulsion de répétition permanente de laquelle il ne sort pas (mythe de Sisyphe selon Camus). On lit aussi une allusion à la transcendance dans la fin du récit Der Mensch erscheint im Holozän. Dans l'image de la quiétude, du silence, du paysage figé depuis la nuit des temps et que la disparition d'un homme (ou tout au moins de sa conscience) n'affecte pas, dans l'image de la chouette, symbole de mort, signe du passage de la vie vers la mort, est évoquée une forme de transcendance en communion avec la nature, avec le Tout, malgré la disparition du vieil homme qu'est Geiser. Nous pensons que « la richesse et l'originalité existentielle de son œuvre de maturité dépasse les limites de la narration du Moi. Dans cet univers littéraire sans Dieu, une forme de transcendance se trouve justement dans la contemplation et la fusion dans l'espace $»^{20}$.

Et l'évocation de la transcendance est aussi présente, en pointillés, dans le Troisième Journal, paru en 2010, qui regroupe des notes de Frisch prises au début des années 1980 (lesdites années newyorkaises). Dans ce journal aux accents pessimistes et désabusés, on trouve beaucoup d'allusions à la disparition d'êtres chers et à la fin de vie, à la mort de proches. Frisch cerne le problème de la mort en tournant autour de sa signification: «Une société qui certes produit la mort comme jamais auparavant, mais une mort sans transcendance; et sans transcendance il n'y a que le présent, pour être plus exact: l'instantanéité de notre existence comme vide face à la mort $\aleph^{21}$. Sa constatation est amère, si la vie matérielle devient de plus en plus facile et la durée de vie plus longue, la vie dans l'instant d'un éternel présent sans transcendance, reste une vie d'interrogation, sans but et sans finalité. Il pose ainsi, dans sa constatation désabusée et de manière implicite, la question de la transcendance. Mais sans jamais vraiment 
donner de réponse à cette question, en tournant autour de la question et en éludant la réponse. Les certitudes existentialistes des années 1950-60 sont loin. Le temps de la vie s'est écoulé, dans la vie tout est une question de temps et nous sommes d'accord avec la constatation d'Emmanuel Levinas :

Comme si le temps, dans sa diachronie, revenait à une éternité manquée, à 'l'image mobile de l'éternité immobile' ou de l'Un consommé. Henri Bergson qui, pour la première fois dans l'histoire des idées, tente de penser le temps en dehors de cet échec de l'éternité, aura, en effet, désigné le destin de cette notion dans la philosophie comme celui d'un devenir passant pour une privation de l'éternitéz ${ }^{2}$.

On retrouve cette privation d'éternité dans les représentations que se fait Frisch d'une vie après la mort, sous la même forme que la vie terrestre (avec peu de variations). L'homme qu'il dessine ainsi se trouve pris, tel Sisyphe, dans un cercle infernal de répétitions de ses scénarios de vie, tel un damné ; et c'est à notre sens, une forme de représentation de l'enfer au sens biblique du terme, qu'on peut lire dans la pièce Triptychon.

28 La question de la mort est récurrente dans toute son œuvre à travers le problème du temps qui est un des facteurs décisifs dans les indices de narration chez Frisch. Dans une quête approfondie et récurrente du sens de la vie, il interroge l'être au monde, sa façon d'être au monde, il le remet en question (cf. Blaise Pascal, se reconnaître). Cette mise en question passe par la faculté de dire Je, de s'engager avec et/ou pour l'Autre, jusqu'à constater les limites de son Moi («Le Moi est haïssable», Pascal) et la décrépitude qui gagne avec l'âge. Déjà dans le roman Stiller, il s'interroge sur le sens de la mort de l'Autre dans la disparition de la compagne, qui débouche sur la solitude de l'être. Pourtant cette mort de l'Autre ne remet pas le personnage en question ${ }^{23}$, la confrontation avec la mort et la perte de sa femme ne lui permet pas de sortir de soi, de se transformer: la capacité de résilience est étroite chez Frisch. S'il inscrit ses personnages masculins, centres des regards et de l'action, dans un monde de solitude, il les place aussi, en homme de son temps, dans un monde sans Dieu, comme s'il choisissait l'altérité à la place de la divinité.

La mort, présente dans chacune de ses œuvres (mort de Faber, de Julika dans Stiller, tentatives de suicide de Stiller, de Felix Schaad, fin du roman Gantenbein, problématique centrale de la pièce Triptychon, etc.), luit comme un fanal, garde-fou lumineux d'une vie bien remplie, bien vécue et bien appréciée. La très belle image de l'oasis dans le désert vient conclure dans le Troisième Journal sa ligne de vie : « La vie comme une oasis. - / la mort tel le désert autour. - / D'où puis-je savoir cela ? ${ }^{24}$ La mort, c'est elle qui donne à la vie sa saveur, comme un point sur un « $\mathrm{i}$ ». Au-delà des œuvres de fiction, l'intérêt et la préoccupation pour la mort transparaît dans les petits écrits en prose et dans ses Journaux. À force d'observer la détérioration de soi, il a une conscience aiguë du problème du vieillissement dans la société et du devenir des personnes âgées, ainsi il anticipe sur la fin de vie en militant pour une mort digne et pour le choix de mourir (cf. « Vereinigung Freitod» dans Tagebuch 1966-1971), tenaillé par une angoisse de sa propre finalité et une obsession de la maladie incurable entretenue depuis longtemps.

30 À la fin de son œuvre ce ne sont plus ses personnages qui profitent de sa plume comme accompagnement vers leur mort, mais sa vie privée. La mort d'un ami devient un sujet proche et brûlant, comme le souligne Emmanuel Levinas à propos de la mort d'autrui :

La mort de l'autre homme me met en cause et en question comme si, de cette mort invisible à l'autre qui s'y expose, je devenais, de par mon éventuelle indifférence, le complice; et comme si, avant même que de lui être voué moi-même, j'avais à 
répondre de cette mort de l'autre, et à ne pas laisser autrui seul à sa solitude mortelle. C'est précisément dans ce rappel de ma responsabilité par le visage, qui m'assigne, qui me demande, qui me réclame, c'est dans cette mise en question qu'autrui est prochain ${ }^{25}$.

31 La crainte pour autrui, pour la disparition d'autrui, se fait sentir dans la vie privée de Frisch et le Troisième Journal (Entwürfe zu einem dritten Tagebuch, 2010) montre un homme soucieux de ses amis dans l'épisode de la maladie et de la disparition de son ami Peter Noll. Il évoque l'annonce faite par son ami de son cancer au téléphone (11), les pensées que son ami lui a dictées sur la mort ("Diktate ÜBER STERBEN UND TOD“, 49), leur voyage en Egypte écourté par une crise qui leur a fait prendre un avion sanitaire le troisième jour ${ }^{26}$, sa mort, alors que Frisch était à New-York (118), sa tombe (101), l'éloge funèbre dont Frisch est chargé par son ami (123) ${ }^{27}$.

Son évocation des morts qui l'entourent et le fait qu'il leur survive lui montre la précarité de la fin qui approche (59). Sa conscience de la proximité de la mort et du fait de vivre avec ses souvenirs et ses morts, est aigüe : «Mon cercle d'amis parmi les morts ne cesse de s'agrandir. Bientôt je serai le plus vieux d'entre vous. Seules ma mère et ma sœur sont encore plus âgées, mon père devient toujours plus jeune que moi ${ }^{28}$. Pourtant il ne fait montre d'aucune angoisse, vivre avec ses morts est un état de faits, ils sont ses compagnons de vieillesse, signes d'un passé disparu: "Les morts sont difficiles, car ils n'ont jamais vu les êtres auxquels je suis lié, Alice par exemple ${ }^{29}$. Il évoque aussi la présence de son frère, disparu avant lui (170), il discute avec lui, comme il le fait avec Tchekhov. Son monde est fait de souvenirs, qui entretiennent sa mémoire. Avec la vieillesse, c'est la solitude qui augmente, compagne des personnes âgées. Si elle est un objet de souci pour les proches ${ }^{30}$, elle n'angoisse pas l'écrivain: «Je fais la vaisselle - / Suis-je seul ? - Je sèche la vaisselle - / Parfois j'aime bien être seul ${ }^{31}$. Mais la conscience de vieillir est vive et sa capacité d'observation acérée, il n'a rien perdu de son sens critique :

Je deviens un vieillard. / On devient un vieillard, quand on ne se sent plus obligé à rien, quand on ne pense pas devoir quelque chose à quelqu'un sur terre, et pour cela on n'a pas besoin de marcher avec une canne ou d'être en fauteuil roulant; il existe aussi des vieillards capables de faire des randonnées ${ }^{32}$.

Même très affutée, la conscience envers le quotidien et les autres, s'émousse et la distance envers la vie et les autres, augmente inéluctablement, il n'y résiste pas: "Avant tout je suis aussi effrayé par ma négligence grandissante envers mes amis, mon indifférence grandissante envers les événements publics, ma liberté grandissante... ${ }^{33}$. Le grand fil directeur de ce Journal est et reste l'idée d'être vieux, qui le poursuit et l'obsède, cette obsession se lit à différents endroits du Journal et elle culmine dans la répétition, qui sonne comme une tentative d'exorcisation: "Je suis vieux, je suis vieux $\aleph^{34}$. Frisch vit très mal son vieillissement physique et les conséquences qu'il a sur sa vie quotidienne et ses relations avec ses proches.

On trouve pourtant dans son œuvre quelques allusions à la divinité. Il a toujours observé une grande discrétion sur ce sujet et se qualifie lui-même d'agnostique et non d'athée, lui qui dans cette position a tenu l'éloge funèbre de son ami Peter Noll : « Vol retour pour Zurich. / Pour honorer ma promesse. / Que peut dire un agnostique à l'église ? $»^{35}$ Le fait de la croyance religieuse et d'une transcendance est évoqué, peutêtre malgré lui, lorsqu'il pense à l'expérience de la mort qu'a faite son ami, qui à présent a une longueur d'avance sur lui : «Au contraire, lui, le mort, a fait entre temps sans nous une expérience à laquelle j'ai encore droit et qui n'est pas transmissible - 
sinon par une révélation dans la foi $»^{36}$. Il fait là aussi état de son rapport au catéchisme, de ce qui le rebute dans le christianisme, de ce à quoi il lui est impossible de croire, mais aussi, et ceci est surprenant, de ce qui l'a marqué : le sermon sur la montagne ${ }^{37}$. Il n'a pas eu la capacité de croire, laisse-t-il entendre.

Pourtant une conscience et une réflexion du moi transcendantal même ténue existe, il évoque, toujours dans le Troisième Journal, dans une vision de la vie après la mort, une maison blanche de treize chambres avec une véranda. Il imagine le paysage alentour, une forêt et des collines, des groupes d'arbres, un lac au loin. La sorte d'arbres dépend de l'endroit où l'on se trouve, il $\mathrm{y}$ a des traces de pas d'animaux dans la neige. Il imagine une cheminée dans la maison, un piano, une bibliothèque, une cuisine, une vie sociale, des amis qui viennent y séjourner. La vie y ressemble à la vraie vie d'ici bas. La représentation de la vie après la mort chez Frisch reste ancrée dans l'immanence.

La question de fond qui n'est jamais abordée de manière directe dans son œuvre, mais qui taraude tous ses protagonistes, est celle de la capacité au bonheur. Les fins de romans sont toutes ouvertes, avec des accents de doute (Stiller seul à Glion), de satisfaction («La vie me plaît », dans Gantenbein), avec un penchant net pour la vie dans l'instant et la recherche du bonheur ici bas. L'expérience de l'amour est toujours une expérience de foi, puisque face à l'inconnu il faut croire à ce qu'on ne connaît pas (encore), à une promesse de bonheur à deux, dont on ne sait pas si elle se réalisera, et c'est une aventure risquée dans laquelle on s'engage (chez Frisch cet engagement débouche de manière quasi systématique sur l'échec et fonctionne comme une compulsion de répétition). Selon Gabriel Marcel, dire à quelqu'un qu'on l'aime, signifie le rendre éternel, car dans ma conscience il ne mourra pas. Gabriel Marcel affirme que c'est par autrui que l'on arrive à soi : c'est aussi le seul moyen pour appréhender l'autre dans son originalité. La vie terrestre se trouve dans des preuves de vie, des rencontres, des visages, des relations interpersonnelles, dans l'altérité, Frisch souscrit entièrement à ce fait, même s'il n'est pas un penseur chrétien comme Marcel. Si Spinoza croit que le bonheur est pour tous, parce que chacun peut accomplir un travail de réflexion, il souhaite une vie dans une société où règnent la liberté d'expression et la tolérance deux siècles plus tard, Frisch rêve encore de ceci, sans le théoriser. Spinoza remplace l'âme par la notion de conscience, on passe ainsi à la conscience du corps, la capacité de connaître et de réfléchir. Un individu a comme essence le désir, selon lui le désir est le moteur de l'individu - dans une recherche métaphysique dans l'ici bas, il met l'accent sur une éthique débarrassée de considérations religieuses. Frisch le rejoint également sur ce point. Si le bonheur est la satisfaction de tous nos penchants selon Kant, il n'est pas une joie constante, mais un "passage " selon Spinoza. Chez Frisch ces passages existent, ce sont les instants où l'être réalise la beauté de la vie, des instants fugaces. Depuis que l'humanité, notamment à travers Nietzsche, réclame un bonheur ici et maintenant sans promesse pour l'au-delà, le concept de la jouissance immédiate lié à l'instantanéité de la vie a fait son chemin. Frisch mesure les limites d'un monde sans transcendance, dont la mort serait un arrêt brutal de la conscience et de la vie. Si la modernité a cru pouvoir réaliser le bonheur de tous dans une forme de paix sociale, Frisch se situe à cette croisée. Dans la postmodernité, le bonheur est défini comme étant toujours possible et une fin en soi. Cette notion contredit la définition du bonheur comme inscrit dans la durée. Frisch se situe plutôt dans l'esprit de Montaigne, qui propose une sagesse de la vie, du quotidien et de l'homme en devenir, il propose d'augmenter la joie et de diminuer la tristesse, car plus que le bonheur, c'est la compréhension d'eux-mêmes que cherchent ses personnages. Dans son univers 
littéraire, on n'échappe pas à son destin, on ne refait pas le monde, on ne parvient pas non plus à le changer, ni à se changer soi, on ne parvient à comprendre ni soi, ni l'autre ; c'est la recherche de soi qui est montrée et mise en avant, une recherche de la compréhension de soi et des autres qui mène au monde. Et s'il est vrai, selon Alain que le bonheur est une récompense qui vient à ceux qui ne l'ont pas cherchée, selon Frisch la sagesse ce n'est pas d'attendre que ce bonheur arrive, mais c'est d'aimer la vie ; il rejoint ainsi les derniers mots de Montaigne dans les Essais : « Pour moi, donc, j'aime la vie. » Il aime la vie, comme elle est : passagère, fluctuante, difficile, ardue, imprévisible. Comme Montaigne, il trouve que la vie ne vaut que par le plaisir qu'on y trouve, Montaigne qui affirme à juste titre : «Il n'est rien si beau et légitimé que de faire bien l'homme et dûment, ni sciences si ardue de bien et naturellement savoir vivre cette vie ; et de nos maladies, la plus sauvage, c'est mépriser notre être.» (III, 13). Frisch partage cette pensée et il ajoute que le bonheur, c'est d'écrire, et c'est l'utopie de l'écriture littéraire qui le guide, il affirme : "Sans utopie, nous serions des êtres sans transcendance $»^{38}$.

La dernière étape de maturation de la pensée de Max Frisch, après l'engagement sur le terrain et les interventions dans des tribunes, marque un tournant philosophique, dans une désillusion depuis le début des années 1980 : une désillusion qui culmine dans le discours incontournable des Journées Littéraires de Soleure en mai 1986: Am Ende der Aufklärung steht das Goldene Kalb ${ }^{39}$, où il constate la dérive par l'argent des sociétés occidentales et de la Suisse avant tout. Il a atteint un temps où l'écriture devient un exercice moins régulier et où le tarissement du flot des idées préfigure la fin de son chemin de vie.

Si le parcours littéraire de Max Frisch est très centré sur la notion d'identité, chère aux années 1950, cette thématique marque son œuvre jusqu'à la maturité dans les années 1960. Dans un face à face avec l'autre féminin et dans une recherche constante du Moi, les personnages de Frisch font preuve de cécité à la vraie vie et aux sentiments et se complaisent dans une incompréhension de l'autre et un refus de s'accepter tels qu'ils sont, en hommes de leur temps. Ils évoluent dans un monde antimétaphysique où la transcendance est absente et où se vérifie l'être à la mort de Heidegger. À travers des fables ou par le biais du mythe, ils cherchent à éviter un destin ordinaire (se marier, avoir des enfants, entre autres) pour échouer dans toutes leurs tentatives de vivre une autre vie hors des sentiers battus; ils se cognent aux limites de la vie, à celles de leur milieu social, de leur entourage et de leur environnement, de leur siècle, de leur propre personnalité et de leurs limites physiques. Pour constater que les préjugés sont là, le racisme, le non-dit, le mensonge, la tromperie et les faux-semblants. Ils veulent rester libres de leurs choix, ne pas s'engager et pouvoir conserver toute latitude de changer de cap, de choix et de chemin de vie, de compagne aussi. Ils veulent dépasser les limites imposées par la vie (ou Dieu) et vivent dans une absence de transcendance. Dans un temps sans dieu et sans mythe, ils sont des héros postmodernes avant l'heure, qui se complaisent parfois dans une pulsion de mort comme refus du monde et de l'existence ; l'œuvre de Frisch propose un démontage du mythe, par exemple celui de Don Juan, et une "recomposition du mythe, vu sous un angle existentialiste et sceptique $\aleph^{40}$. Frisch parvient à faire descendre le mythe au niveau de la vie quotidienne et domestique (voir la fin de Don Juan), tout comme ses allusions de la philosophie existentialiste sont appliquées à la vie de ses personnages (notamment à Stiller), pour finalement constater que la philosophie n'est pas transposable dans la vie et que ses recettes ne marchent pas (ni Kierkegaard, ni Heidegger, ni Sartre). À travers l'Autre 
(sur le tard, il s'agit surtout de l'amitié), la mise en marche de l'interrogation de l'audelà de la vie terrestre, de l'être après la mort, rapproche les thématiques frischiennes de l'altérité philosophique (dont Levinas, Spinoza et surtout Montaigne), interrogation permanente du regard de l'Autre, et en finalité de la transcendance, vers laquelle tend l'être pour la mort qu'est l'homme, du fond de son angoisse existentielle.

Pourtant les fins des œuvres insistent sur l'attachement à la vie, malgré ses vicissitudes, malgré les succès en demi teinte de ses héros, malgré les défaites et l'échec des rébellions de ses héros (Don Juan, Stiller, Homo faber, etc.). Frisch montre l'échec de l'homme quand il tente de sortir de sa condition, des moules sociaux qui sont faits et décidés pour lui par la société dans laquelle il vit. Il dénonce tout ce qui entrave la liberté de choix et montre avec ironie et parfois une certaine cruauté, que le choix est bien le mode trompeur par excellence, celui auquel l'homme veut croire, alors que le jeu est joué d'avance. Tardivement, dans un mouvement de désespoir et de résignation, le message de l'œuvre tardive propose d'accepter la place que l'homme occupe dans la chaîne de la vie (cf. Der Mensch erscheint im Holozän).

Max Frisch le rebelle garde cependant sa morale : elle est anti-contrainte par définition, il ne voulait pas mettre les personnes dans des moules pour ne pas être mis dans un moule ou classifié à son tour. Il y a donc bien une conscience morale chez Frisch sans édiction de sentences morales et l'homme est ce qui est en question dans sa morale. L'œuvre tardive montre la difficulté de supporter l'autre, le quotidien, de se supporter soi, son corps vieillissant. Pour cet épicurien conscient qu'il faut profiter des instants qui nous sont donnés, ce grand amoureux des femmes et vivant dans la crainte de l'impuissance (cf. Montauk), la vieillesse, la dégénérescence, la décrépitude et la mort étaient ses pires ennemis. S'il fut un homme jamais satisfait de ce qu'il avait, malgré les nombreuses récompenses, les importants succès littéraires et le soutien artistique dont il bénéficia, il resta jusqu'au bout un homme soucieux de sa postérité, des traces qu'il laissera et du devenir de son œuvre et de l'humanité.

\section{NOTES}

1. Voir l'affirmation de Max Frisch à ce sujet : "12. ich bin kein Philosoph", in Ich schreibe für Leser. Antworten auf vorgestellte Fragen (1964), Gesammelte Werke, Band V, Frankfurt am Main, Suhrkamp, 1998, p. 327.

2. Max Frisch, Tagebuch 1946-1949, Frankfurt am Main, Bibliothek Suhrkamp, 1974, p. 37. Cité d'après Journal 1946-1949, traduit de l'allemand par Madeleine Besson et Philippe Pilliod, Paris, Gallimard 1964, p. 33.

3. Voir Ronald Laing, The divided Self. An existential study in Sanity and Madness. London, 1960. Allusion est faite ici à la version allemande: Das geteilte Selbst : eine existentielle Studie über geistige Gesundheit und Wahnsinn Reinbeck bei Hamburg, Rowohlt, 1977, p. 40.

4. Max Frisch, Stiller, traduit par Éliane Kaufholz-Messmer, Paris, Grasset, 1991, p. 133. 
5. Søren Kierkegaard, Post-scriptum définitif et non scientifique aux miettes philosophiques, Trad. de Paul-Henri Tisseau et Else-Marie Jacquet-Tisseau, in Euvres complètes, Paris, Édition de l'Orante, 1977, p. 22.

6. Voir à cet effet le développement très érudit de Willy Michel et Edith Michel, «Pour comprendre Max Frisch », in Régine Battiston-Zuliani et Philippe Forget, Relire Max Frisch. Les chemins de l'identité, Paris, Les Éditions du Temps, 2001, p. 59-77.

7. "[...] ich komme nämlich nicht von der Literatur, sondern von der Eigenerfahrung her und würde, wenn man das Wort nicht missbrauchen will, mich $\mathrm{zu}$ der Gattung der Notwehrschriftsteller rechnen." Heinz Ludwig Arnold, Schriftsteller im Gespräch I, Zürich, Haffmans, 1990, p. 252. (Traduction de l'auteur).

8. Lorsqu'on consulte dans les archives Max Frisch (ETH Zürich) les tapuscrits (parfois de nombreuses versions) de ses romans, il est évident à quel point il a travaillé et retravaillé la forme de ses textes, leur agencement et la succession des différents scénarios. Rien n'est laissé au hasard, la preuve se trouve là, que Frisch voulait tout maitriser et calculer, mettre en scène (jusqu'à superviser la mise en scène de ses pièces de théâtre). Le degré de réflexion (et parfois de commentaire) est frappant chez lui.

9. La parabole est une histoire souvent courte, qui illustre une morale ou une doctrine philosophique par des exemples tirés du quotidien. Son but est d'être un exercice de démonstration par comparaison. La morale et la religion se servent de cette figure de narration et la littérature aussi en est remplie.

10. Voir par exemple, Homo faber, in Max Frisch, Gesammelte Werke, Bd. 4, Frankfurt am Main, Suhrkamp, 1998, p.117, où Faber évite de creuser l'évidence de sa relation de paternité envers Sabeth. Voir aussi à propos du non-dit dans les dialogues dans l'œuvre de Frisch notre étude : Régine Battiston, Funktion der Dialoge in Max Frischs Romanen Stiller und Mein Name sei Gantenbein, in Anne Betten und Monika Dannerer, Dialogue Analysis IX : Dialogue in Literature and the Media, Tübingen, Niemeyer, 2005, p. 159-172.

11. Voir entre autres Régine Battiston et Edith Michel, «Homo faber: rapport d'une mort annoncée ", in Régine Battiston et Philippe Forget, Relire Max Frisch, Paris, Les Éditions du Temps, 2001, p. 101-122, ici p. 105-108.

12. "Als der Sohn fünfundfünfzig war, sagte meine Mutter nicht ohne Strenge : du solltest nicht immer über Frauen schreiben, denn du verstehst sie nicht", Max Frisch, Montauk, Gesammelte Werke, Bd. 6,Frankfurt am Main, Suhrkamp, 1998, p. 688.

13. Fabien Chareix, article sur l'existence, in Dictionnaire des concepts philosophiques sous la direction de Michel Blay, Larousse, CNRS Éditions, Paris, 2007, p. 302.

14. Voir notamment Edith et Willy Michel: "Pour mieux comprendre Max Frisch ", in Régine Battiston-Zuliani et Philippe Forget, Relire Max Frisch. Les chemins de l'identité, op. cit., 1999, p. 59-77.

15. Régine Battiston: "Don Juan et Barbe Bleue ou le rapport ou féminin selon Max Frisch», in Florence Le Diagon-Jacquin: Don Juan, Le Paon d'Hera, Gazette Interdisciplinaire Thématique Internationale $\mathrm{n}^{\circ} 7$, Éditions du Murmure, 2010, p. 193-209, ici p. 208.

16. C'est aussi ce que reproche la mère de Frisch à son fils qui reproduit ce reproche dans Montauk (voir note 12). On notera cette mise en écho de notions fondamentales que Frisch opère dans sa propre œuvre.

17. Max Frisch, Blaubart, Gesammelte Werke, Bd. 7, Frankfurt am Main, Suhrkamp, 1998, p. 334.

18. "Wörter - alles Sehen wandelte sich bei ihm in Wörter. Alles Hören wurde zum Wort. Seine Wörter haben Gerüche. Sie sind fassbar - tastbar. Alle seine Sinne waren auf das Wort gerichtet. Er dachte in Wörtern. Er hatte Respekt vor dem Wort. Er prüfte bei jedem Wort Eigenheit und Sinn. Er kannte seine Wörter. Er hat mit ihnen und durch sie gelebt." Gottfried Honegger: 
"Nachruf auf Max Frisch“, in Widerstand aus Verantwortung, Kapitel "Wortwahrheit Nachruf auf Max Frisch“, Zürich, Limmatverlag, 1991, p. 53-56, ici p. 53. (Traduction de l'auteur). 19. Voir notamment le fameux discours : "Rede zur Verleihung des Friedenspreises des deutschen Buchhandels 1976": "Wir hoffen“" (GW, VII, 7-19, 1976).

20. Régine Battiston, Lectures de l'identité narrative, Max Frisch, Ingeborg Bachmann, Marlen Haushofer, W.G. Sebald. Paris, Éditions Orizons, 2009, p. 280.

21. "Eine Gesellschaft, die zwar Tod produziert wie noch nie, aber Tod ohne Transzendenz, und ohne Transzendenz gibt es nur die Gegenwart, richtiger gesagt: die Augenblicklichkeit unserer Existenz als Leere vor dem Tod“. Max Frisch, Entwürfe zu einem dritten Tagebuch, Hg. Peter von Matt, Berlin, Suhrkamp, 2010, p. 73. (Traduction de l'auteur).

22. Emmanuel Levinas, "L'autre transcendance ", in Altérité et transcendance, Paris, Fata Morgana, 1995, p. 36.

23. Cf. « La mort de l'Autre qui me met en question... ", ibid., p. 45.

24. "Leben als Oase. - / der Tod als die Wüste ringsum - / Woher will ich das wissen ?"Max Frisch, Entwürfe zu einem dritten Tagebuch, op. cit., p. 20. (Traduction de l'auteur).

25. Emmanuel Levinas, "L'autre transcendance", in Altérité et transcendance, op. cit., p. 45.

26. Max Frisch, Entwürfe zu einem dritten Tagebuch, op. cit., p. 55-57 et 62-65.

27. "Voir le discours reproduit dans les œuvres complètes : "Rede zum Tod von Peter Noll“, in GW, Band VII, op. cit., p. 74-79 (1982).

28. "Immer größer wird mein Freundeskreis unter den Toten. Bald bin ich der älteste unter Euch. Nur die Mutter und die Schwester sind immer noch älter, mein Vater wird immer jünger als ich.“ Ibid., p. 59. (Traduction de l'auteur).

29. "Die Toten sind schwierig, weil sie die Menschen, denen ich verbunden bin, nie gesehen haben, Alice zum Beispiel."Ibid., p. 60. (Traduction de l'auteur).

30. "Als ich sie später [...] die Strasse hinunter begleite, hängt sie ihren Arm in meinen Arm und möchte wissen, ob ich mich einsam fühle. "Ibid., p. 59.

31. "Ich spüle das Geschirr - / Bin ich einsam? / Ich trockne das Geschirr - / Manchmal bin ich gern allein.“ Ibid., p. 60. (Traduction de l'auteur).

32. "Ich werde ein Greis. / Man wird ein Greis, wenn man sich zu nichts mehr verpflichtet fühlt, wenn man nicht meint, irgendjemand in der Welt irgendetwas zu schulden, und dazu braucht einer noch nicht am Stock zu gehen oder im Rollstuhl zu sitzen; es gibt auch wanderfähige Greise." Ibid., p. 85. (Traduction de l'auteur).

33. "Vorderhand erschreckt mich noch meine zunehmende Nachlässigkeit gegenüber Freunden, meine zunehmende Gleichgültigkeit gegenüber öffentlichen Ereignissen, meine zunehmende Freiheit..." Ibidem.(Traduction de l'auteur).

34. "Ich bin alt, ich bin alt." Ibid., p. 137.

35. "Flug nach Zürich zurück. / Um das Versprechen einzulösen./ Was hat in der Kirche ein Agnostiker zu sagen ?" Ibid., p. 121.(Traduction de l'auteur).

36. "Er hingegen, der Verstorbene, hat inzwischen eine Erfahrung gemacht ohne uns, die Erfahrung, die mir erst noch bevorsteht und die sich nicht vermitteln lässt - es geschähe denn durch eine Offenbarung im Glauben." Ibid., p. 123.(Traduction de l'auteur).

37. Ibid., p. 124.

38. "Ohne Utopie wären wir Lebewesen ohne Transzendenz." Max Frisch, "Wir hoffen." Rede zur Verleihung des Friedenspreises des deutschen Buchhandels 1976, in GW, Band VII, op. cit., p. 12. (Traduction de l'auteur).

39. Max Frisch, Am Ende der Aufklärung steht das Goldene Kalb, Sankt Gallen, Erker Verlag, 1991. 
40. Régine Battiston: "Don Juan et Barbe Bleue ou le rapport ou féminin selon Max Frisch », in Florence Le Diagon-Jacquin : Don Juan, Le Paon d'Hera, op. cit., p. 193-209, ici p. 204.

\section{RÉSUMÉS}

Cette contribution montre le développement dans l'œuvre de Max Frisch des trois concepts : existence, altérité et transcendance, qui marquent trois stades de son évolution. Elle se pose aussi la question de la présence de la philosophie dans cette œuvre qui amorce son développement sur le terrain de l'existentialisme à partir du concept phare de l'identité (narrative). L'œuvre de la maturité montre une ouverture vers l'altérité autre que féminine, dans un engagement citoyen face aux aléas du monde des années 1970-1980; la littérature de Frisch prend alors un ton critique et engagé contre les injustices. L'œuvre tardive laisse transparaître, à côté de cet engagement, une interrogation sur le vieillissement et la mort, en présentant de fugaces traces de transcendance, en dehors du sentier religieux. Son œuvre reste ancrée dans le présent de son temps par un engagement permanent pour le devenir de l'homme à travers l'écriture.

Dieser Beitrag zeigt die Entwicklung in Max Frischs Werk der drei Konzepte Existenz, Alterität und Transzendenz, welche drei Stadien seiner Entwicklung darstellen. Er wirft auch die Frage der Präsenz der Philosophie in diesem Werk auf, das sich auf dem Boden des Existenzialismus durch das bedeutende Identitätskonzept entwickelt. Das Werk der Reife wendet sich der nicht mehr nur weiblichen Alterität zu, in einem bürgerlichen Engagement gegen die Unruhen der Welt der siebziger-achtziger Jahre; Frischs Literatur weist nun einen kritischen und engagierten Ton gegen die Ungerechtigkeit auf. Im Spätwerk wird neben diesem Engagement eine Fragestellung über Altern und Tod auch durch flüchtige Spuren der Transzendenz außerhalb des religiösen Weges deutlich. Sein Werk schreibt sich in die Gegenwart seiner Zeit mit dem permanenten Engagement für die Menschenzukunft durch das Schreiben ein.

This contribution shows the development of three concepts, all of which mark three stages in the evolution of in the works of Max Frisch : existence, otherness and transcendence. This paper also raises the question of the presence of philosophy in this work, which starts its development on the ground of existentialism from the flagship concept of (narrative) identity. The work of maturity shows openness to non feminine otherness - in a civic commitment against the vagaries of the world in the 1970's-1980's; Frisch's literature takes a critical and engaged tone against injustice. In addition to this commitment, his late work shows a questioning about oldness and death, by presenting fleeting traces of transcendence, outside of a religious path. His work is thus rooted in the present of his time in an ongoing commitment for humanity's fate through writing.

\section{INDEX}

oeuvrecitee Trosième Journal 


\section{AUTEURS}

\section{RÉGINE BATTISTON}

Institut de recherche en langues et littératures européennes, Université de Haute-Alsace 